\title{
Combination of optical coherence tomography (OCT) and OCT angiography increases diagnostic efficacy of Parkinson's disease
}

\author{
Jing Zou ${ }^{1,2 \#}$, Kangcheng Liu ${ }^{1,2 \#}$, Fangling $\mathrm{Li}^{1,2}, \mathrm{Yi} \mathrm{Xu}^{1,2}$, Lu Shen ${ }^{3}$, Huizhuo Xu${ }^{1,2}$ \\ ${ }^{1}$ Eye Center of Xiangya Hospital, Central South University, Changsha, China; ${ }^{2}$ Hunan Key Laboratory of Ophthalmology, Changsha, China; \\ ${ }^{3}$ Department of Neurology, Xiangya Hospital, Central South University, Changsha, China
}

"These authors contributed equally to this work.

Correspondence to: Huizhuo Xu. Eye Center of Xiangya Hospital, Central South University, No.87, Xiangya Road, Kaifu District, Changsha 410008, China. Email: xhz1030@csu.edu.cn.

Background: To assess the diagnostic efficacy of optical coherence tomography (OCT) and OCT angiography (OCTA) in Parkinson's disease (PD).

Methods: OCT was used to obtain macular parameters and peripapillary retinal nerve fiber layer (RNFL) thickness. The macular superficial retinal vessel and foveal avascular zone (FAZ) were quantified with OCTA. The area under the receiver operating characteristic curve (AUC) indicated the diagnostic efficacy of the parameters.

Results: Thirty-five eyes from 35 PD patients and 35 eyes from 35 age-matched healthy subjects who served as controls were evaluated. The mean RNFL thickness overall and the thicknesses of the other three quadrants were similar in $\mathrm{PD}$ patients compared with controls $(\mathrm{P} \geq 0.358)$. The RNFL thickness at the temporal quadrant, total macular volume (TMV), macular retinal thickness (MRT), and ganglion cell-inner plexiform layer complex (GCL-IPL) thickness were reduced in the eyes of $\mathrm{PD}$ patients $(\mathrm{P} \leq 0.046)$. There was no difference between the CMT of PD patients compared with control subjects $(\mathrm{P}=0.163)$. The vessel length density (VLD) in the central, inner and full regions; vessel perfusion density (VPD) in all regions; and the FAZ circularity index in PD patients were significantly lower than in controls $(\mathrm{P} \leq 0.049)$. The AUC of the VLD in PD in the central, inner and full regions were 0.712, 0.728, and 0.650, respectively; The VPD in the central, inner and full region were $0.711,0.756$, and 0.682 , respectively. The mean RNFL thickness in the temporal quadrant, TMV and MRT revealed an AUC of 0.718, 0.693 and 0.699, respectively. The VPD in the outer region, FAZ circularity and GCL-IPL thickness did not have diagnostic ability in distinguishing PD from normal eyes $(\mathrm{P} \geq 0.05)$. The AUCs of a combination of the VLD in the inner region and TMV, the VLD in the inner region and MRT, the VPD in the inner region and TMV, and the VPD in the inner region and MRT, were $0.843,0.849,0.849$, and 0.848 , respectively $(\mathrm{P} \leq 0.001)$.

Conclusions: Decreased OCT and OCTA parameters were detected in the eyes of PD patients. Combined non-invasive measurements of OCT and OCTA had better diagnostic ability than either alone, and may provide an additional biomarker for PD progression.

Keywords: Parkinson's disease (PD); optical coherence tomography (OCT); OCT angiography (OCTA); diagnostic efficacy

Submitted Mar 19, 2020. Accepted for publication Jun 10, 2020.

doi: 10.21037/qims-20-460

View this article at: http://dx.doi.org/10.21037/qims-20-460 


\section{Introduction}

Parkinson's disease (PD) is a frequently observed neurodegenerative disorder that features motor symptoms, including bradykinesia, rest tremor, rigidity, and postural instability, and non-motor symptoms, such as sleep disorders, anosmia, depression, cognitive impairment and vision deficits (1-3). The aggregation of misfolded protein alpha-synuclein (mAS) is the main cause of injury to dopaminergic (DA) neurons in the central nervous system (4). Postmortem studies have documented the presence of $\mathrm{mAS}$ in retinal cells and hypothesized the retinal structure changes that meditate vision deficits including altered visual acuity, decreased contrast sensitivity, photophobia, and complex visual hallucinations $(5,6)$.

Optical coherence tomography (OCT) has been used for more than 10 years to detect and quantify changes in the retina in PD (7). OCT assessment can assist with delineating the pathogenesis and determining a diagnosis $(8,9)$. Interestingly, small vessel pathology has been described as a key factor involved in the progression of $\mathrm{PD}$ and the retina could be a window to the brain for examining microvascular components (10). Thus, assessment of the retinal microvasculature can generate a better understanding of the pathogenesis of $\mathrm{PD}$. OCT angiography (OCTA) is a rapidly developing technology, and it has been used in various studies of ophthalmic diseases such as glaucoma, diabetic retinopathy, and epiretinal membranes (11-13). In addition to retinal vessel density, OCTA-based foveal avascular zone (FAZ) metrics is used to explore the perifoveal capillary network in disease associated with vascular impairment. Some groups reported macular vessel density (VD) and FAZ metrics could be potential biomarkers in the clinical evaluation of diseases, such as glaucoma, and diabetic retinopathy (14-16). Recently, several studies have demonstrated reduced macular VD in patients with PD and suggested OCTA with quantitative assessment could be an additional biomarker for detecting vessel changes in the early stages of PD $(17,18)$. However, there are a few studies that have reported the utility of OCT and OCTA parameters. The purpose of our study was to evaluate the diagnostic efficiency of OCT and OCTA parameters and determine whether the combination of these measurements is a useful detection method for PD progression.

\section{Methods}

\section{Subjects}

This was a cross-sectional study and all research methods followed the Helsinki Declaration (as revised in 2013). After approval by the Medical Ethics Committee of Xiangya Hospital (No. 202002030), all subjects signed an informed consent form according to the principles of ethics.

Patients with sporadic PD were recruited from the outpatient neurology clinics of Xiangya Hospital and were referred to the Department of Ophthalmology at Xiangya Hospital. Each patient underwent a detailed clinical interview and a complete neurological examination. PD was diagnosed by two experienced neurologists according to the United Kingdom PD Brain Bank Criteria (19). Disease severity in $\mathrm{PD}$ patients was rated by one trained neurologist using the Hoehn and Yahr (H\&Y) scale (20).

A total of 35 eyes from $35 \mathrm{PD}$ patients and 35 eyes from 35 age-matched healthy controls were selected randomly and enrolled from January 2019 to December 2019 in the Ophthalmology Department of Xiangya Hospital. A complete ophthalmologic examination, including best corrected visual acuity (BCVA), intraocular pressure measurement, slit lamp microscopy, dilated fundus examination with a 90-diopter lens, and OCT and OCTA examinations, was performed after patients were diagnosed with PD in the Neurology Department.

The subjects were excluded if they met the following criteria: (I) BCVA less than 0.5 evaluated with a Snellen chart; (II) ophthalmic diseases, such as glaucoma, fundus diseases, and corneal diseases; (III) presence of retinal or optic nerve diseases; (IV) diopter spherical power $>6.00$ $6.00 \mathrm{D}$ and/or astigmatism $>3.00-3.00 \mathrm{D}$; (V) intraocular pressure higher than $21 \mathrm{mmHg}$; (VI) history of ocular trauma or intraocular surgery, history of hypertension or diabetes, and family history of glaucoma.

\section{OCT measurements}

SD-OCT (Cirrus; Carl Zeiss Meditec Inc., Dublin, CA, USA) was used to obtain the macular parameters and peripapillary RNFL in all subjects (8). A retinal area of $6.0 \mathrm{~mm}$ horizontal and $6.0 \mathrm{~mm}$ vertical were scanned in a Macular Cube $512 \times 128$ mode to measure the central macular thickness (CMT), macular retinal thickness (MRT), total macular volume (TMV), and average ganglion cell-inner plexiform layer complex (GCL-IPL) with an automatic analysis algorithm. A 3.64-mm diameter circle around the optic dis was scanned and the RNFL protocol was used to measure the average peripapillary RNFL thickness and the average RNFL thicknesses in the temporal, nasal, superior and inferior quadrants. 


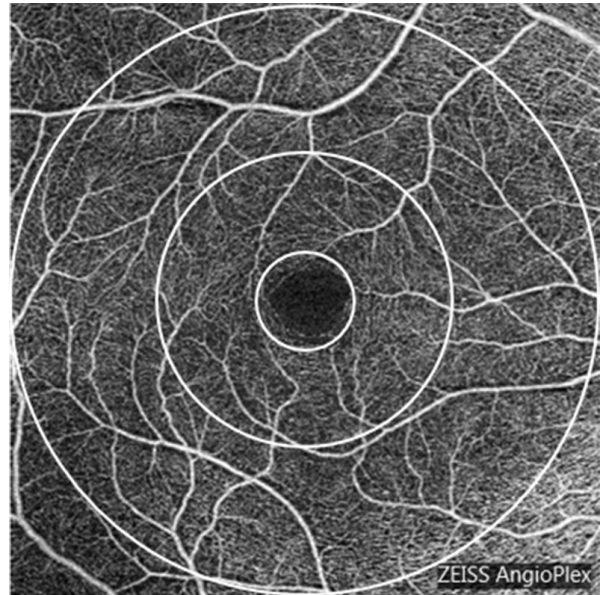

Figure 1 Macular area measurement map. The central area is the center region. The inner, outer and full regions were calculated separately at various distances from the fovea: inner $(1-3 \mathrm{~mm}$ diameter region), outer (3-6 $\mathrm{mm}$ diameter region) and full $(6 \mathrm{~mm}$ diameter region).

\section{OCTA measurements}

All subjects underwent SD-OCTA examination using the AngioPlexTM OCTA system (Cirrus; Zeiss, Dublin, USA; software version 10.0.0.14618) (21). OCTA obtained a $6 \times 6 \mathrm{~mm}$ volumetric macular superficial retinal vessel image that consisted of vessels from the layer of the inner limiting membrane (ILM) to the inner plexiform layer (IPL). Poorquality scans with a strength index less than seven were excluded. The vessel length density (VLD, defined as the total length of the perfused vasculature) and vessel perfusion density (VPD, defined as the total area of the perfused vasculature per unit area in a region of measurement) were calculated separately at various distances from the fovea: central ( $1 \mathrm{~mm}$ diameter region), inner (1-3 $\mathrm{mm}$ diameter region), outer (3-6 mm diameter region) and full $(6 \mathrm{~mm}$ diameter region; Figure 1) (22). The FAZ indicators of the macular area, including area, perimeter and circularity index, were automatically calculated by the system software (Cirrus; Zeiss, Dublin, USA; software version 10.0.0.14618). The FAZ circularity index is a measure of the shape of the FAZ, where 1 indicates a circular shape and 0 indicates an irregular shape. By observing the parameters of FAZ, damage to the capillaries in the macular arch area could be assessed (15).

\section{Statistical analysis}

Statistical analyses were performed with SPSS version 22.0
(IBM Corp, Armonk, NY, USA). The normal distribution of the data was tested with the Kolmogorov-Smirnov test. Therefore, the data were presented as the mean \pm standard deviation (range) of the Gaussian distribution value and the median (95\% confidence interval) of the non-Gaussian distribution. The comparisons of OCT parameters and OCTA metrics between PD patients and healthy controls were analyzed with an LSD $t$-test for Gaussian distribution values and a Tamhane's T2 test for non-Gaussian distribution values. A $\mathrm{P}$ value less than 0.05 was considered statistically significant. The area under the receiver operating characteristic (AUC) was determined to evaluate the discrimination capability of single OCT and OCTA parameters, and combined OCT and OCTA parameters.

\section{Results}

This study comprised 35 eyes from 35 patients and 35 eyes from 35 healthy control subjects. The clinical features and ophthalmologic characteristic are listed in Table 1. There were no statistically significant differences in age, sex, BCVA and IOP $(P \geq 0.263)$. The mean disease duration was $3.2 \pm 2.0$ years, and the median $H \& Y$ stage was 2 . Among these patients, 16 patients were in $\mathrm{H} \& \mathrm{Y}$ stage 1,15 patients were $\mathrm{H} \& \mathrm{Y}$ in stage 2 , and 4 patients were in $\mathrm{H} \& \mathrm{Y}$ stage 3 .

Table 2 presents the OCT and OCTA parameters for all subjects. Except for the RNFL thickness of the temporal quadrant being thinner $(\mathrm{P}=0.002)$, the mean RNFL thickness overall and the thicknesses of the other three quadrants were similar in PD patients compared with controls $(\mathrm{P} \geq 0.358)$. The TMV, MRT and GCL-IPL thicknesses were reduced in eyes with $\mathrm{PD}$ compared with control eyes ( $\mathrm{P}=0.005, \mathrm{P}=0.008$, and $\mathrm{P}=0.046$, respectively). There was no difference between the CMT of PD patients compared with control subjects.

Regarding the macular microvascularity, most of the OCTA parameters showed differences between the two groups. Compared with the eyes of healthy controls, the VLD of eyes in PD patients was significantly decreased in the central, inner and full regions, with statistically significant differences $(\mathrm{P}=0.002, \mathrm{P}<0.001$, and $\mathrm{P}=0.018)$; However, there was no significant change in the outer region $(\mathrm{P}=0.138)$. VPD in the eyes of $\mathrm{PD}$ patients was significantly reduced in all regions compared to control eyes, with statistically significant differences $(\mathrm{P} \leq 0.049)$. For the FAZ index, there were no differences in the FAZ area and perimeter between the two groups $(\mathrm{P}=0.320$, and $\mathrm{P}=0.117$, respectively). In the eyes of $\mathrm{PD}$ patients, the value 
Table 1 Clinical features and parameters of the participants

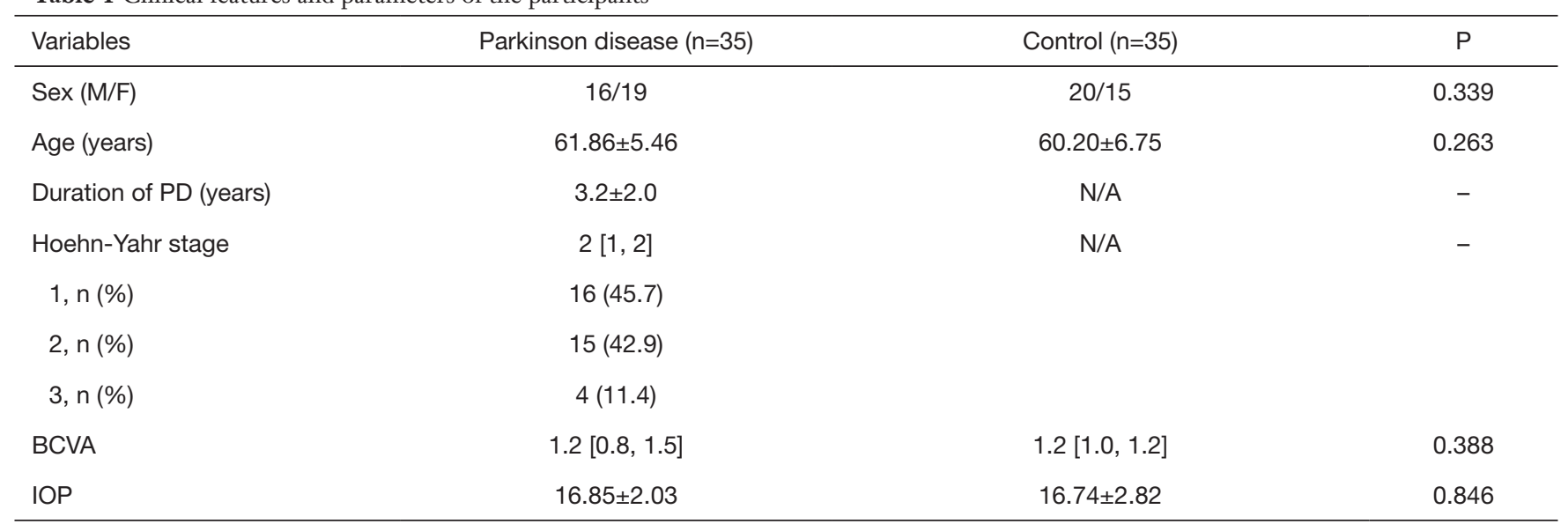

PD, Parkinson's disease; BCVA, best corrected visual acuity; IOP, intraocular pressure; N/A, not available; SD, standard deviation.

of the FAZ circularity index was lower than that of the control eyes $(\mathrm{P}=0.037)$.

Different OCT and OCTA partition parameter values were used to analyze the ROC curve and calculate the AUC to evaluate the accuracy of detection. The AUC of the VLD in PD in the central, inner and full regions were 0.712 , 0.728 , and 0.650 , respectively (Table 3, Figure $2 A$ ). The VPD in the central, inner and full region were $0.711,0.756$, and 0.682 , respectively (Table 3, Figure $2 A$ ). The mean RNFL thickness in the temporal quadrant, TMV and MRT revealed an AUC of 0.718, 0.693 and 0.699, respectively (Table 3, Figure 2B). The VPD in the outer region, FAZ circularity and GCL-IPL thickness did not have diagnostic ability in distinguishing PD from normal eyes $(\mathrm{P}>0.05)$ (Table 3).

Values of the AUC between 0.6 and 0.7 are considered to indicate a weak predictive capacity, values between 0.71 and 0.8 have moderate predictive capacity, and values greater than 0.8 have strong predictive capacity (23). Therefore, we calculated and listed the high value, which was greater than 0.8 , of the combinations of OCT and OCTA parameters in Figure 2. The AUCs of the VLD in the inner region combined with TMV and MRT were 0.843 , and 0.849 , respectively (Figure 2C,D). The AUCs of a combination of the VPD in the inner region, and TMV and the VPD in the inner region and MRT to detect a PD diagnosis were 0.849 , and 0.848 , respectively (Figure 2E,F).

\section{Discussion}

Recent studies have demonstrated retinal degeneration in $\mathrm{PD}$, but there is a limited number of studies on the diagnostic ability of the retinal structure related index. In this study, we confirmed changes of the macular and peripapillary retinal structure in patients with $\mathrm{PD}$ using both OCT and OCTA. Subsequently, the utility of OCT and OCTA parameters as diagnostic markers were explored separately and in combination. In addition, we evidenced the OCT-plus-OCTA combination could be a better biomarker for detecting PD progression, over OCT or OCTA alone.

OCT was applied to detect retinal structural changes that were associated with PD for more than a decade, which enabled capturing the in-vivo retinal image (9). Although there were no standards for OCT parameter values in PD, because of differences in methodology, OCT equipment, ethnicity and the age of the population in different studies, OCT examination was regarded as a potential tool for early diagnosis and prognosis in PD (24). In our study, the decline in the RNFL thickness in the temporal quadrant, TMV, MRT and GCL-IPL thickness was consistent with recent reports that were conducted with the same SD-OCT devices $(8,25,26)$. Ganglia cells are DA cells, and increased apoptosis of ganglion cells with glutamate and neurotrophin deprivation could lead to GCL-IPL thinning in PD (27). The RNFL comprises axons of the ganglion cells, and the thicknesses of the four quadrants are different (28). Hence, the temporal quadrant was more sensitive to the damage of DA cells in progressive $\mathrm{PD}$, which may explain how the RNFL thickness becomes thinner at the temporal quadrants. As for the anatomy of the macular region, the nerve fibers and ganglion cells are absent in the fovea (29). 
Table 2 OCT and OCTA parameters of the participants

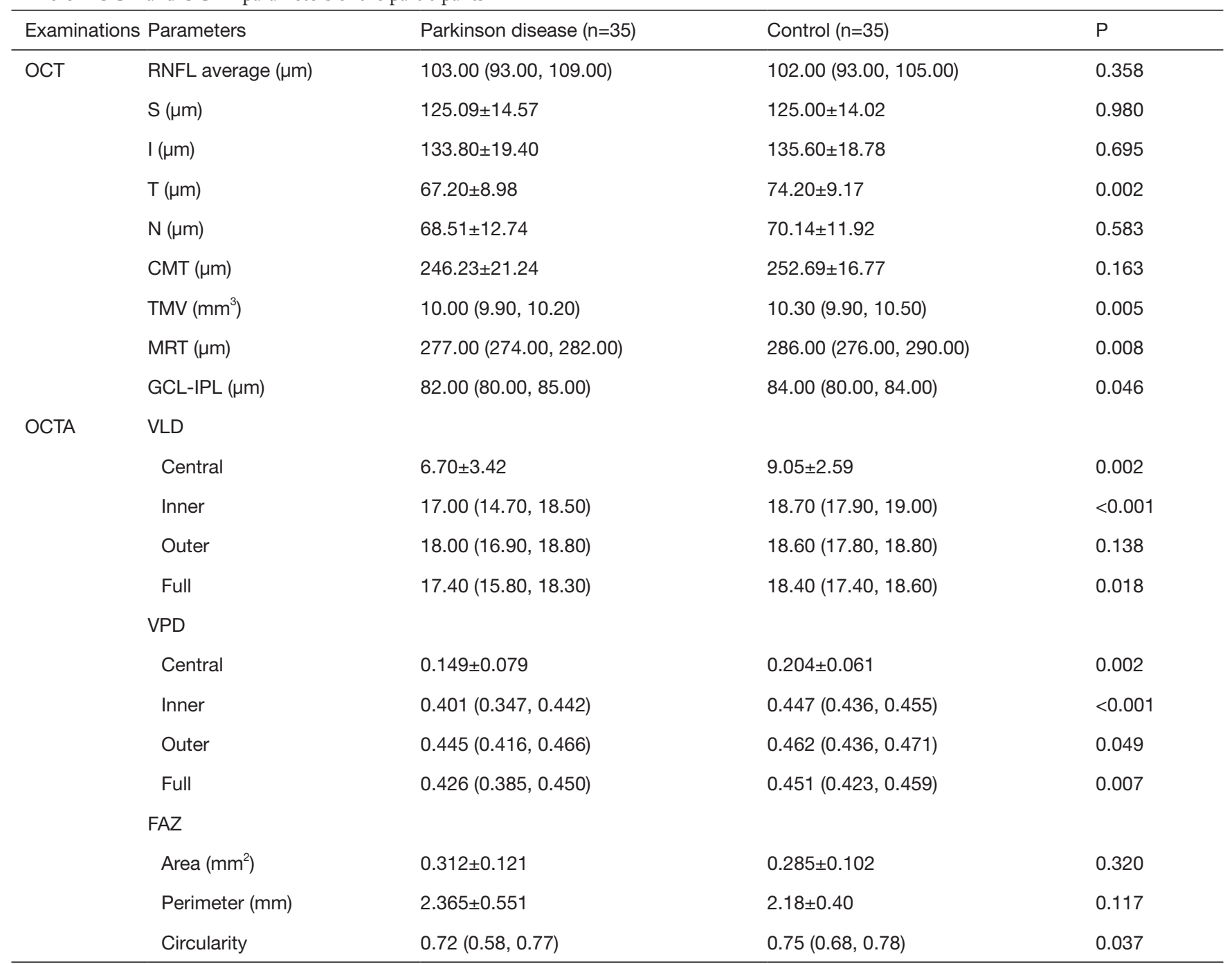

OCT, optical coherence tomography; OCTA, optical coherence tomography angiography; RNFL, retinal nerve fiber layer; S, superior quadrant; I, inferior quadrant; T, temporal quadrant; N, nasal quadrant; CMT, central macular thickness; TMV, total macular volume; MRT, macular retinal thickness; GCL-IPL, ganglion cell-inner plexiform layer complex; VLD, vessel length density; VPD, vessel perfusion density; FAZ, foveal avascular zone.

Thus, the reduction of retinal thickness appeared unpronounced in the central region, while the TMV, MRT were clearly thinner in PD.

There were a few researches focused on the changes of vascular morphology in $\mathrm{PD}$, and they reported the increased string vessels, decreased and shorten capillaries in multiple brain regions $(30,31)$. As extensions of the brain, the retinal and cerebral microvasculature share common characteristics (32). Therefore, the retina provides a novel direction method to study vascular degeneration which is regarded as an important contributing factor to PD progression. To date, a number of studies have indicated a significant decrease in the retinal VD of neurodegenerative diseases compared to normal controls, such as Alzheimer's disease, multiple sclerosis and PD, using OCTA (33).

Oxygen consumption in the macular is greater than in other tissues in the body, and the vascular supply of the macula is only a single-layered parafoveal capillary arcade (34). These evidences imply us to pay attention to the macular retinal VD because the macula is more sensitive to hypoxia and ischemia (29). In addition to retinal vessel density, OCTA-based FAZ metrics enabled exploration of the 
Table 3 The areas under the ROC curve for results

\begin{tabular}{|c|c|c|c|}
\hline Parameters & \multicolumn{3}{|c|}{ PD vs. Ctr } \\
\hline VLD central & 0.712 & $0.589-0.835$ & 0.002 \\
\hline VLD inner & 0.728 & $0.607-0.849$ & 0.001 \\
\hline VLD full & 0.650 & $0.518-0.783$ & 0.031 \\
\hline VPD inner & 0.756 & $0.638-0.873$ & $<0.001$ \\
\hline VPD outer & 0.618 & $0.487-0.750$ & 0.089 \\
\hline VPD full & 0.682 & $0.557-0.808$ & 0.009 \\
\hline FAZ circularity & 0.636 & $0.506-0.766$ & 0.050 \\
\hline MRT & 0.699 & $0.576-0.823$ & 0.004 \\
\hline GCL-IPL & 0.625 & $0.493-0.757$ & 0.072 \\
\hline
\end{tabular}

PD, Parkinson's disease; Ctr, control; AUC, area under the ROC curve; ROC, receiver-operating characteristic; VLD, vessel length density; VPD, vessel perfusion density; FAZ, foveal avascular zone; RNFL, retinal nerve fiber layer; TMV, total macular volume; MRT, macular retinal thickness; GCL-IPL, ganglion cell-inner plexiform layer complex.

perifoveal capillary network in diseases associated with vascular impairment (35). Recently, Kwapong et al. (17) reported impairment of the macular retinal microvascular and suggested OCTA measurements of the retinal microvascular could be an objective tool for early diagnosis of PD. In our study, we observed the superficial retinal vessels in different macular regions, including the fovea, parafovea and perifovea vessels that were contained in the central $(1 \mathrm{~mm})$, inner $(1-3 \mathrm{~mm})$ and outer $(3-6 \mathrm{~mm})$ rings, respectively (36). VD was described with different OCTA metrics including VLD (37), the blood flow index (38), micro vessel density (39) and fractal dimension (40) in previous studies. Here, we propose use of VLD and VPD, which have high repeatability and reproducibility $(21,41)$, would provide a more accurate reflection of macular vascular perfusion status. The VLDs of the eyes in PD patients were significantly decreased in the central, inner and full regions, but not in the outer region. Although the VPDs in the eyes of PD patients were reduced in all regions compared with control eyes, the differences in the perifovea vessels were slight $(\mathrm{P}=0.049)$. As for the FAZ index, there was no difference in the FAZ area and perimeter; however, the value of FAZ circularity index was lower than that of control eyes, which indicated injury of the alignment of the parafoveal capillary arcade. Considering all results, we hypothesize microcirculatory alterations in the eyes of PD patients occurred in the fovea and parafovea, but not the perifovea.

Although major studies have supported OCT and OCTA as methods that can provide new parameters to consider in a PD diagnosis, sufficient data are lacking to describe the utility of retinal structure and vascularity factors in the progression of PD. In our study, the AUCs of mean RNFL thickness in the temporal quadrant, TMV and MRT were 0.718, 0.693 and 0.699, while the AUCs of the VLD and VPD in the central, inner and full regions were $0.712,0.711,0.728,0.756,0.650$, and 0.682 , respectively. We found the diagnostic ability of OCT and OCTA parameters to be comparable. The moderate diagnostic capacities of these parameters drove us to identify a more effective combination index. Interestingly, higher values for the AUC could be achieved through the combination of VLD in the inner region and TMV or MRT, and VPD in the inner region and TMV or MRT. OCT and OCTA parameters were closely associated with the progression of pathology in PD, and they are more convenient to obtain than neuroimaging indicators. Diagnosis of PD is now based on the appearance of typical symptoms, and the diagnostic accuracy of the criterion is $82 \%$ (42). Therefore, the combination of ophthalmic indicators will be of value in 

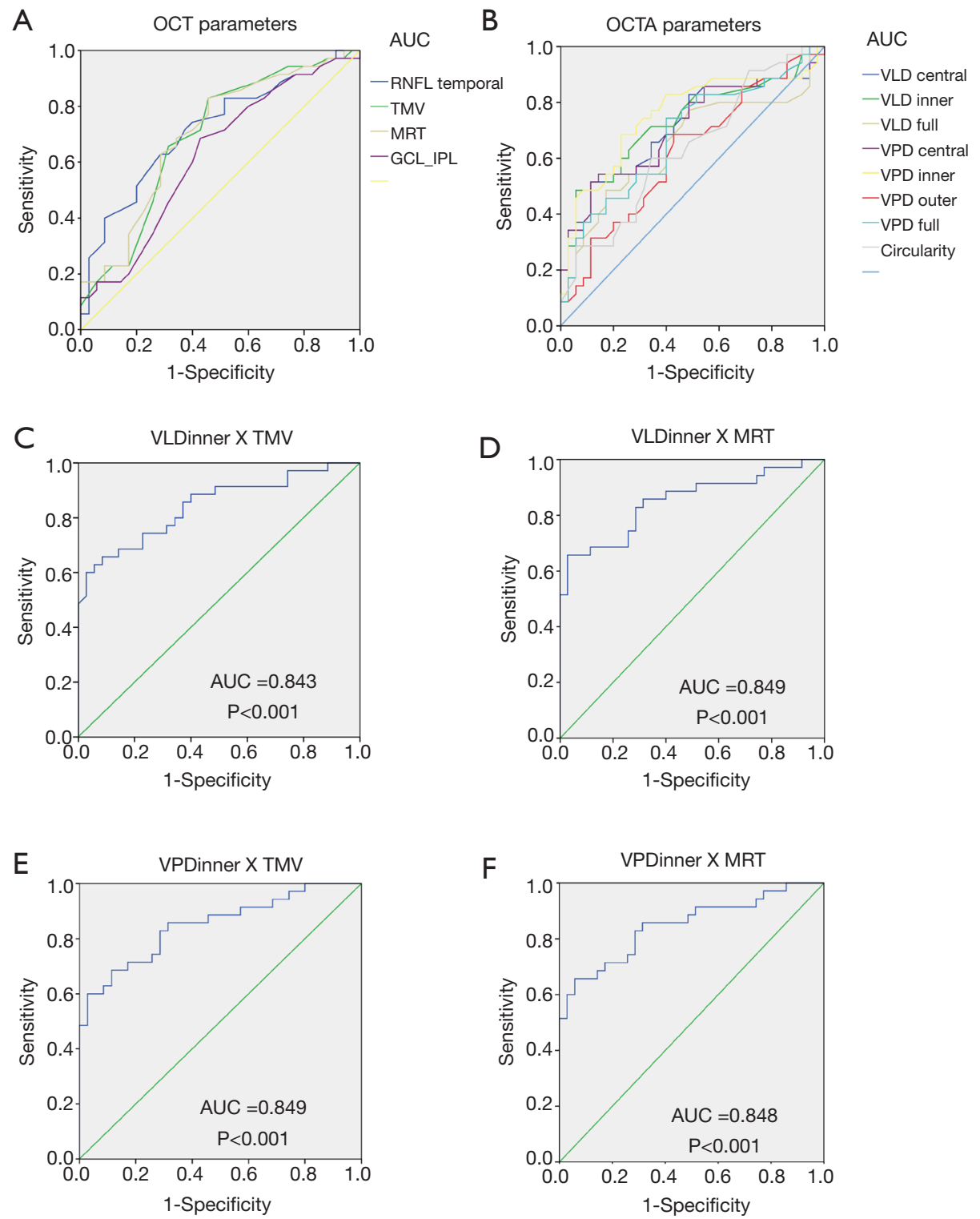

Figure 2 ROC curve analysis of the OCT and OCTA parameters for discriminating PD patients from healthy controls. (A) ROC curve analysis of single OCTA parameters; (B) ROC curve analysis of single OCT parameters; (C) ROC curve of the combined assessment of VLD inner and TMV was 0.843 (95\% CI: 0.750-0.936); (D) ROC curve of the combined assessment of VLD inner and MRT was 0.849 (95\% CI: 0.757-0.941); (E) ROC curve of the combined assessment of VPD inner and TMV was 0.849 (95\% CI: 0.759-0.939); (F) ROC curve of the combined assessment of VPD inner and MRT was 0.848 (95\% CI: 0.757-0.940). OCT, optical coherence tomography; OCTA, OCT angiography; PD, Parkinson's disease; AUC, area under the ROC curve; ROC, receiver-operating characteristic; VLD, vessel length density; VPD, vessel perfusion density; RNFL, retinal nerve fiber layer; GCL-IPL, ganglion cell-inner plexiform layer complex; TMV, total macular volume; MRT, macular retinal thickness.

practical applications for the diagnosis of PD.

There are some limitations in our study. First, this was a retrospective study and each group had a relatively small sample of 35 eyes. Most PD patients recruited for our study were in the early stage of disease development. Further studies with larger sample sizes and patients in all stages are needed to explore the diagnostic value of OCT and OCTA indicators for PD. Second, we did not evaluate the visual 
functions of our PD patients. Past researches suggested abnormal results of VEP which measure the integrity of the visual pathway, insensitive electrical activity of multifocal electroretinography (ERG) which is specific for measuring retinal function, and decreased contrast sensitivity in PD (26). Thus, the visual acuity being similar between PD and controls does not indicate visual functional is unimpaired. Besides, Huang et al. (43) reported the AUC of combined amplitude density of P1 with macular volume was 0.901 . Therefore, in the further study we would evaluate visual functions that may serve as additional indices for ophthalmic combination indicators. Third, some PD patients had been treated with levodopa and the influence from this medicine is unknown.

\section{Conclusions}

The eyes of PD patients showed a progressive reduction in the RNFL thickness, GCL-IPL thickness, TMV, MRT, macular VD, as well as the FAZ circularity index. These indicators had a weak-moderate diagnostic capacity, but the combined use of OCT and OCTA indicators improved both sensitivity and specificity. Hence, non-invasive OCT and OCTA technology could provide a more accurate combination clinical diagnostic biomarker in the assessment of PD progression.

\section{Acknowledgments}

Funding: This study was supported by a grant from the Hunan Natural Science Foundation (No. 2019JJ40528) to Huizhuo Xu and a grant from the National Natural Science Foundation of China (No. 81600713) to Jing Zou.

\section{Footnote}

Conflicts of Interest: All authors have completed the ICMJE uniform disclosure form (available at http://dx.doi. org/10.21037/qims-20-460). The authors have no conflicts of interest to declare.

Ethical Statement: The study was approved by the Medical Ethics Committee of Xiangya Hospital (No. 202002030), all subjects signed an informed consent form according to the principles of ethics.

Open Access Statement: This is an Open Access article distributed in accordance with the Creative Commons
Attribution-NonCommercial-NoDerivs 4.0 International License (CC BY-NC-ND 4.0), which permits the noncommercial replication and distribution of the article with the strict proviso that no changes or edits are made and the original work is properly cited (including links to both the formal publication through the relevant DOI and the license). See: https://creativecommons.org/licenses/by-nc-nd/4.0/.

\section{References}

1. Salawu FK, Danburam A, Olokoba AB. Non-motor symptoms of Parkinson's disease: diagnosis and management. Niger J Med 2010;19:126-31.

2. Jankovic J. Parkinson's disease: clinical features and diagnosis. J Neurol Neurosurg Psychiatry 2008;79:368-76.

3. Xu R, Hu X, Jiang X, Zhang Y, Wang J, Zeng X. Longitudinal volume changes of hippocampal subfields and cognitive decline in Parkinson's disease. Quant Imaging Med Surg 2020;10:220-32.

4. Karpinar DP, Balija MB, Kugler S, Opazo F, RezaeiGhaleh N, Wender N, Kim HY, Taschenberger G, Falkenburger BH, Heise H, Kumar A, Riedel D, Fichtner L, Voigt A, Braus GH, Giller K, Becker S, Herzig A, Baldus M, Jackle H, Eimer S, Schulz JB, Griesinger C, Zweckstetter M. Pre-fibrillar alpha-synuclein variants with impaired beta-structure increase neurotoxicity in Parkinson's disease models. EMBO J 2009;28:3256-68.

5. Bodis-Wollner I, Kozlowski PB, Glazman S, Miri S. alphasynuclein in the inner retina in Parkinson disease. Ann Neurol 2014;75:964-6.

6. Armstrong RA. Visual symptoms in Parkinson's disease. Parkinsons Dis 2011;2011:908306.

7. Inzelberg R, Ramirez JA, Nisipeanu P, Ophir A. Retinal nerve fiber layer thinning in Parkinson disease. Vision Res 2004;44:2793-7.

8. Ma LJ, Xu LL, Mao CJ, Fu Y'T, Ji XY, Shen Y, Chen J, Yang YP, Liu CF. Progressive Changes in the Retinal Structure of Patients with Parkinson's Disease. J Parkinsons Dis 2018;8:85-92.

9. Mailankody P, Lenka A, Pal PK. The role of Optical Coherence Tomography in Parkinsonism: A critical review. J Neurol Sci 2019;403:67-74.

10. Bohnen NI, Muller ML. Cerebral small vessel disease and incident parkinsonism: the RUN DMC study. Neurology 2016;86:1268-9.

11. Chen CL, Wang RK. Optical coherence tomography based angiography [Invited]. Biomed Opt Express 2017;8:1056-82.

12. Kashani AH, Chen CL, Gahm JK, Zheng F, Richter 
GM, Rosenfeld PJ, Shi Y, Wang RK. Optical coherence tomography angiography: A comprehensive review of current methods and clinical applications. Prog Retin Eye Res 2017;60:66-100.

13. Zhang Q, Rezaei KA, Saraf SS, Chu Z, Wang F, Wang RK. Ultra-wide optical coherence tomography angiography in diabetic retinopathy. Quant Imaging Med Surg 2018;8:743-53.

14. Choi J, Kwon J, Shin JW, Lee J, Lee S, Kook MS. Quantitative optical coherence tomography angiography of macular vascular structure and foveal avascular zone in glaucoma. PLoS One 2017;12:e0184948.

15. Kwon J, Choi J, Shin JW, Lee J, Kook MS. Alterations of the Foveal Avascular Zone Measured by Optical Coherence Tomography Angiography in Glaucoma Patients With Central Visual Field Defects. Invest Ophthalmol Vis Sci 2017;58:1637-45.

16. Lu Y, Simonett JM, Wang J, Zhang M, Hwang T, Hagag AM, Huang D, Li D, Jia Y. Evaluation of Automatically Quantified Foveal Avascular Zone Metrics for Diagnosis of Diabetic Retinopathy Using Optical Coherence Tomography Angiography. Invest Ophthalmol Vis Sci 2018;59:2212-21.

17. Kwapong WR, Ye H, Peng C, Zhuang X, Wang J, Shen M, Lu F. Retinal Microvascular Impairment in the Early Stages of Parkinson's Disease. Invest Ophthalmol Vis Sci 2018;59:4115-22.

18. Shi C, Chen Y, Kwapong WR, Tong Q, Wu S, Zhou Y, Miao H, Shen M, Ye H. Characterization by fractal dimension analysis of the retinal capillary network in Parkinson disease. Retina 2020;40:1483-91.

19. Hughes AJ, Daniel SE, Kilford L, Lees AJ. Accuracy of clinical diagnosis of idiopathic Parkinson's disease: a clinico-pathological study of 100 cases. J Neurol Neurosurg Psychiatry 1992;55:181-4.

20. Goetz CG, Poewe W, Rascol O, Sampaio C, Stebbins GT, Counsell C, Giladi N, Holloway RG, Moore CG, Wenning GK, Yahr MD, Seidl L. Movement Disorder Society Task Force report on the Hoehn and Yahr staging scale: status and recommendations. Mov Disord 2004;19:1020-8.

21. Lei J, Durbin MK, Shi Y, Uji A, Balasubramanian S, Baghdasaryan E, Al-Sheikh M, Sadda SR. Repeatability and Reproducibility of Superficial Macular Retinal Vessel Density Measurements Using Optical Coherence Tomography Angiography En Face Images. JAMA Ophthalmol 2017;135:1092-98.

22. Chu Z, Lin J, Gao C, Xin C, Zhang Q, Chen CL, Roisman
L, Gregori G, Rosenfeld PJ, Wang RK. Quantitative assessment of the retinal microvasculature using optical coherence tomography angiography. J Biomed Opt 2016;21:66008.

23. Gasparini G, Bonoldi E, Viale G, Verderio P, Boracchi P, Panizzoni GA, Radaelli U, Di Bacco A, Guglielmi RB, Bevilacqua P. Prognostic and predictive value of tumour angiogenesis in ovarian carcinomas. Int J Cancer 1996;69:205-11.

24. Chrysou A, Jansonius NM, van Laar T. Retinal layers in Parkinson's disease: A meta-analysis of spectral-domain optical coherence tomography studies. Parkinsonism Relat Disord 2019;64:40-9.

25. Matlach J, Wagner M, Malzahn U, Gobel W. Repeatability of peripapillary retinal nerve fiber layer and inner retinal thickness among two spectral domain optical coherence tomography devices. Invest Ophthalmol Vis Sci 2014;55:6536-46.

26. Kaur M, Saxena R, Singh D, Behari M, Sharma P, Menon V. Correlation Between Structural and Functional Retinal Changes in Parkinson Disease. J Neuroophthalmol 2015;35:254-8.

27. Bodis-Wollner I. Visual deficits related to dopamine deficiency in experimental animals and Parkinson's disease patients. Trends Neurosci 1990;13:296-302.

28. Bendschneider D, Tornow RP, Horn FK, Laemmer R, Roessler CW, Juenemann AG, Kruse FE, Mardin CY. Retinal nerve fiber layer thickness in normals measured by spectral domain OCT. J Glaucoma 2010;19:475-82.

29. Schmidt-Erfurth U, Kohnen T. Encyclopedia of Ophthalmology. Springer Science+Business Media, 2018.

30. Guan J, Pavlovic D, Dalkie N, Waldvogel HJ, O'Carroll SJ, Green CR, Nicholson LF. Vascular degeneration in Parkinson's disease. Brain Pathol 2013;23:154-64.

31. Yang P, Pavlovic D, Waldvogel H, Dragunow M, Synek B, Turner C, Faull R, Guan J. String Vessel Formation is Increased in the Brain of Parkinson Disease. J Parkinsons Dis 2015;5:821-36.

32. Wylęgała A. Principles of OCTA and Applications in Clinical Neurology. Curr Neurol Neurosci Rep 2018;18:96.

33. Wang L, Murphy O, Caldito NG, Calabresi PA, Saidha S. Emerging Applications of Optical Coherence Tomography Angiography (OCTA) in neurological research. Eye Vis (Lond) 2018;5:11.

34. Yu DY, Cringle SJ, Yu PK, Balaratnasingam C, Mehnert A, Sarunic MV, An D, Su EN. Retinal capillary perfusion: Spatial and temporal heterogeneity. Prog Retin Eye Res 
2019;70:23-54.

35. Kwon J, Choi J, Shin JW, Lee J, Kook MS. An Optical Coherence Tomography Angiography Study of the Relationship Between Foveal Avascular Zone Size and Retinal Vessel Density. Invest Ophthalmol Vis Sci 2018;59:4143-53.

36. Arichika S, Hangai M, Yoshimura N. Correlation between thickening of the inner and outer retina and visual acuity in patients with epiretinal membrane. Retina 2010;30:503-8.

37. Agemy SA, Scripsema NK, Shah CM, Chui T, Garcia PM, Lee JG, Gentile RC, Hsiao YS, Zhou Q, Ko T, Rosen RB. Retinal vascular perfusion density mapping using optical coherence tomography angiography in normals and diabetic retinopathy patients. Retina 2015;35:2353-63.

38. Chen CL, Zhang A, Bojikian KD, Wen JC, Zhang Q, Xin C, Mudumbai RC, Johnstone MA, Chen PP, Wang RK. Peripapillary Retinal Nerve Fiber Layer Vascular Microcirculation in Glaucoma Using Optical Coherence Tomography-Based Microangiography. Invest Ophthalmol Vis Sci 2016;57:OCT475-85.

39. Kim JS, Kim YK, Baek SU, Ha A, Kim YW, Jeoung JW, Park $\mathrm{KH}$. Topographic correlation between macular superficial

Cite this article as: Zou J, Liu K, Li F, Xu Y, Shen L, Xu H. Combination of optical coherence tomography (OCT) and OCT angiography increases diagnostic efficacy of Parkinson's disease. Quant Imaging Med Surg 2020;10(10):1930-1939. doi: 10.21037/qims-20-460 microvessel density and ganglion cell-inner plexiform layer thickness in glaucoma-suspect and early normal-tension glaucoma. Br J Ophthalmol 2020;104:104-9.

40. Kim AY, Chu Z, Shahidzadeh A, Wang RK, Puliafito CA, Kashani AH. Quantifying Microvascular Density and Morphology in Diabetic Retinopathy Using SpectralDomain Optical Coherence Tomography Angiography. Invest Ophthalmol Vis Sci 2016;57:OCT362-70.

41. Chang R, Chu Z, Burkemper B, Lee GC, Fard A, Durbin MK, Reznik A, Xu BY, Kashani A, Varma R, Wang RK, Richter GM. Effect of Scan Size on Glaucoma Diagnostic Performance Using OCT Angiography En Face Images of the Radial Peripapillary Capillaries. J Glaucoma 2019;28:465-72.

42. Marsili L, Rizzo G, Colosimo C. Diagnostic Criteria for Parkinson's Disease: From James Parkinson to the Concept of Prodromal Disease. Front Neurol 2018;9:156.

43. Huang J, Li Y, Xiao J, Zhang Q, Xu G, Wu G, Liu T, Luo W. Combination of Multifocal Electroretinogram and Spectral-Domain OCT Can Increase Diagnostic Efficacy of Parkinson's Disease. Parkinsons Dis 2018;2018:4163239. 